\title{
METHOD OF OBTAINING APPROXIMATE FORMULAS
}

\author{
Konstantin Ludanov \\ Institute for Renewable Energy NAS of Ukraine \\ $20 A$ Gnata Hotkevicha str., Kyiv, Ukraine, 02094 \\ k.i.ludanov@ukr.net
}

\begin{abstract}
The two-parameter method of approximating the sum of a power series in terms of its first three terms of the expansion, which allows one to obtain analytic approximations of various functions, decomposes into a Maclaurin series. As an approximation function of this approximation, it is proposed to use elementary functions constructed in the Nth degree, but with a "compressed" or "stretched" variable $\mathrm{x}$ due to the introduction of the numerical factor $\mathrm{M}(\mathrm{x} \equiv \varepsilon \cdot \mathrm{m}, \mathrm{M} \neq 0)$ into it. The use of this method makes it possible to significantly increase the range of very accurate approximation of the obtained approximate function with respect to a similar range of the output fragment of a series of three terms. Expressions for both the approximation parameters ( $\mathrm{M}$ and $\mathrm{N}$ ) are obtained in a general form and are determined by the coefficients of the second and third terms of the Maclaurin series. Also expressions of both approximation parameters are found for the case if the basis function and the approximant function decompose into the Maclaurin series in even powers of the argument. A number of examples of approximation of functions on the basis of the analysis of power series into which they decompose are given.
\end{abstract}

Keywords: approximation of functions, power series expansion, Maclaurin series, sum of a power series, two-parameter approximation, Newton's binomial.

\section{Introduction}

In the analysis of fundamentally important regularities, from which our understanding of new phenomena develops, the role of analytical methods remains extremely high. Their role is also high in the qualitative determination of the parameters of complex physical phenomena, noted in [1]. Therefore, various methods of perturbation theory are so important, which are the main analytical tool for investigating nonlinear physical and technical problems. It is usually possible to calculate only a few terms of the perturbed decomposition, usually no more than two or three. The resulting rows often converge slowly or even diverge. Nevertheless, these several members contain significant information, from which it is necessary to extract all that is possible, is summarized in [2].

How important is the approximation method, which approximates not only the values of the approximating function-basis, but its derivatives, is noted by Russian mathematicians [3] who believe that in the case of an analytically given function it is often useful to replace a too complicated mathematical expression with a simpler one, the basic properties of the function are preserved. Indeed, when approximation of functions is important, not only the accuracy of the approximation, but also the form and properties of the approximating function is important. This is due to the fact that often the approximating function must correspond to the physics of the investigated process. A more accurate reflection of the physics of the process can often be achieved by approximating the function together with the derivatives. Approximating approximations with a small number of parameters have become most widespread in practical investigations. Such expressions are more easily amenable to physical interpretation [3].

Thus, it is very important to develop new methods for reconstructing unknown functions from small fragments of power series into which they decompose, as well as to search for simple approximations for complex functions based on the use of their decompositions into Maclaurin series. Such methods will make it possible to significantly increase the range of their exact approximation, and also to be able to interpret experimental data within the framework of linear dependence in the event that the variable in a complex function is implicitly expressed.

\section{Literature review and problem statement}

Historically, the first method of accelerating the convergence of power series is the fractional-rational transformation of a variable (small parameter) in the form of an Euler transformation: 
$\mathbf{x}=\varepsilon /(1+\varepsilon),[2]$. The goal of the Euler transformation is the transfer of the singularity $\varepsilon=-1$ to the point at infinity. Moreover, if there are no other singularities in the complex plane, then the radius of convergence of the series becomes infinite [2, 4].

From the low-parametric methods of approximation in mechanics, the so-called Shanks "nonlinear transformation" is rather well known. It consists precisely in approximating the sum of the power series in terms of its first three terms. The application of the Shanks nonlinear transformation to the first three terms of the power series $\sum=1+\mathrm{a} \varepsilon+\mathrm{b} \varepsilon^{2}+\ldots$ gives a simple rational fraction

$$
\sum=\left[\mathrm{a}+\left(\mathrm{a}^{2}-\mathrm{b}\right) \varepsilon\right] /(\mathrm{a}-\mathrm{b} \varepsilon),
$$

which is often a more accurate approximation to the sum of the series than its fragment representing the sum of the original three terms. For example, this sum gives an exact value if the series is a geometric progression (both convergent and divergent) [4].

The problem of maximizing the extraction of such information is usually solved by methods of approximating the sum of a power series (Maclaurin) by analytic functions with respect to the coefficients of the first terms of the expansion. The most powerful method of approximation is the Padé method [5-7], in which it is carried out in the framework of approximation by rational functions. However, in many cases [8], the Padé approximation method is unacceptable in principle. First, when as a basis for approximation it is necessary to use non-rational, and other functions, for example, trigonometric. Or when in the solution by the method of a small parameter only a few terms of the expansion are obtained, the coefficients of which must then receive a certain physical interpretation.

In cases when the formula approximating the exact expression or the fragment of the power series into which this expression decomposes, it is sufficient to coincide only the first two derivatives of this function, the most optimal solution is the use of two-parameter approximations of the sum of the Maclaurin series.

In this sense, the most promising method for approximating the sum of a power series in terms of its first three terms is the Ludanov method [9], which abstract (9B 832 DEP) was published in the RZh "Matematika" more than thirty years ago (in No. 9, 1984). A method is developed for two-parameter approximation of the power series of Maclaurin with respect to its first three terms in the form of the Nth power of an arbitrary elementary function $\mathrm{y}(\mathrm{x})$ in which the variable $\mathrm{x}$ is written as the product of the argument $\varepsilon$ by the parameter $\mathbf{M}(\mathrm{x}=\mathbf{M} \varepsilon)$.

\section{Methods of research}

To solve this problem, a powerful method such as the theory of power series was used in this paper. According to Euler [4], there are an infinite number of functions, each of which has an excellent expression for the power series into which it decomposes. Mathematical operations on functions are equivalent to operations on their power series. Analysis of the combination of two mathematical operations on power series: the construction of the Maclaurin series in the Nth power and the introduction of the factor in the composition of its variable $(\mathrm{x}=\mathbf{M} \varepsilon)$, showed the possibility of obtaining an identical decomposition (in fact of some function) of an identical fragment from the first three terms of the series of the elementary function thus modified. This mathematical fact formed the basis for the development of a new method for obtaining approximate formulas, which in the presence of many advantages has a significant drawback: a not very large range of exact approximation. But in many practical cases this approximation accuracy is quite sufficient.

\section{Research results}

4. 1. Approximation of functions that decompose into a power series in the variable $x$

Let's consider the general case when, as a result of solving a nonlinear problem, a fragment of the Maclaurin series is obtained:

$$
\sum \mathrm{c}_{\mathrm{i}} \varepsilon^{\mathrm{i}}=1+\mathrm{a} \varepsilon+\mathrm{b} \varepsilon^{2}+\ldots
$$


the sum of which should be approximated as the Nth power of the function $f(x)$ (where $x=\mathbf{M} \cdot \varepsilon$ ), chosen on the basis of additional considerations (for example, in accordance with the physics of the described phenomenon).

The expansion of the function $\mathbf{y}=\mathrm{f}^{\mathrm{N}}$ in the Maclaurin series has the following form:

$$
\mathbf{y}(\mathrm{x})=1+\mathbf{y}_{(0)}^{\prime} \frac{\mathrm{x}}{1 !}+\mathbf{y}_{(0)}^{\prime \prime} \frac{\mathrm{x}^{2}}{2 !}+\ldots
$$
function $\mathrm{f}_{(\mathrm{x})}$

Let's express the derivatives $\mathbf{y}_{(0)}^{\prime} \mathbf{y}^{\prime \prime}{ }_{(0)}$ in terms of the corresponding derivatives of the chosen

$$
\begin{gathered}
\mathbf{y}_{(0)}^{\prime}=\mathbf{N} \cdot \mathrm{f}_{(0)}^{\mathrm{N}-1} \cdot \mathrm{f}_{(\mathrm{x})}^{\prime}, \\
\mathbf{y}_{(0)}^{\prime \prime}=\mathbf{N} \cdot \mathrm{f}_{(0)}^{\mathrm{N}-2} \cdot\left[(\mathrm{N}-1)\left(\mathrm{f}_{(\mathrm{x})}^{\prime}\right)^{2}+\mathrm{f}_{(\mathrm{x})} \mathrm{f}_{(\mathrm{x})}^{\prime \prime}\right]
\end{gathered}
$$

At the point of expansion $\mathrm{x}=0$ for (3) let's obtain:

$$
\begin{gathered}
\mathbf{y}_{(0)}^{\prime}=\mathrm{N} \cdot \mathrm{f}_{(\mathrm{x})}^{\prime}, \\
\mathbf{y}_{(0)}^{\prime \prime}=\mathrm{N} \cdot\left[(\mathrm{N}-1) \cdot\left(\mathrm{f}_{(0)}^{\prime}\right)^{2}+\mathrm{f}_{(0)}^{\prime \prime}\right] .
\end{gathered}
$$

Taking into account the fact that $\mathrm{x}=\mathrm{M} \varepsilon$, the series (2) can be rewritten as:

$$
\mathrm{f}_{(\mathrm{M} \varepsilon)}^{\mathrm{N}}=1+\mathrm{N} \cdot \mathrm{f}_{(0)}^{\prime} \cdot \mathrm{M} \frac{\varepsilon}{1 !}+\mathrm{N} \cdot\left[(\mathrm{N}-1) \cdot\left(\mathrm{f}_{(0)}^{\prime}\right)^{2}+\mathrm{f}_{(0)}^{\prime \prime}\right] \cdot \mathrm{M}^{2} \frac{\varepsilon^{2}}{2 !}+\ldots
$$

Equating the coefficients of the corresponding powers of the variable $\varepsilon$ for the series (1) and (5), let's obtain a system of two equations with two unknowns:

$$
\left\{\begin{array}{l}
\mathrm{a}=\mathrm{M} \cdot \mathrm{N} \cdot \mathrm{f}_{(0)}^{\prime}, \\
\mathrm{b}=\left(\mathrm{M}^{2} \cdot \mathrm{N} / 2\right) \cdot\left[(\mathrm{N}-1) \cdot\left(\mathrm{f}_{(0)}^{\prime}\right)^{2}+\mathrm{f}_{(0)}^{\prime \prime}\right] .
\end{array}\right.
$$

Solving the obtained system of equations for the unknown parameters $\mathrm{M}$ and $\mathrm{N}$, we obtain their expressions through the known coefficients a and $b$ of the series (1) and the derivatives of the function $f_{(x)}$ chosen as the basis for the approximation $f^{\prime}(x)$ and $f^{\prime \prime}(x)$ in point $(0,1)$ :

$$
\begin{gathered}
\mathrm{N}=\left(\mathrm{a} / \mathrm{f}_{(0)}^{\prime}\right)^{2} \cdot\left[\mathrm{f}^{\prime \prime}{ }_{(0)}-\left(\mathrm{f}_{(0)}^{\prime}\right)^{2}\right] /\left(2 \mathrm{~b}-\mathrm{a}^{2}\right) ; \\
\mathrm{M}=\left(\mathrm{f}_{(0)}^{\prime} / \mathrm{a}\right) \cdot\left(2 \mathrm{~b}-\mathrm{a}^{2}\right) /\left[\mathrm{f}^{\prime \prime}{ }_{(0)}-\left(\mathrm{f}_{(0)}^{\prime}\right)^{2}\right] .
\end{gathered}
$$

A particularly simple expression for the parameters $\mathrm{M}$ and $\mathrm{N}$ is in the case when the function $\mathrm{f}(\mathrm{x})$ is used as the function $\mathrm{f}(\mathrm{x})=1+\mathrm{x}$. In addition, the function $\mathrm{y}(\mathrm{x})$ is obviously expressed in the form of a Newton binomial $y(x)=(1+x)^{\mathrm{N}}$.

The first and second derivatives in this case take the values $\mathrm{f}(\mathrm{x})=1, \mathrm{f}(\mathrm{x})=0$. And the approximation parameters take the following values:

$$
M=a \cdot\left(1-2 b / a^{2}\right), N=\left(1-2 b / a^{2}\right)^{-1}
$$

The same values of the parameters $\mathrm{M}$ and $\mathrm{N}$ can also be obtained directly, i. e. equating the coefficients $a$ and $b$ of the series (1) to the coefficients for the corresponding powers of the binomial series:

$$
(1+\mathrm{M} \cdot \varepsilon)^{\mathrm{N}}=1+\mathrm{N} \cdot \mathrm{M} \cdot \frac{\varepsilon}{1 !}+\mathrm{N} \cdot(\mathrm{N}-1) \cdot \mathrm{M}^{2} \cdot \frac{\varepsilon^{2}}{2 !}+\ldots
$$


Here, it should be noted that if the series (1) is a geometric progression (for $b=a^{2}$ ), then the approximation of this sum in the Newton binomial for $\mathrm{y}=(1+\mathrm{M} \cdot \varepsilon)^{\mathrm{N}}$ gives its exact value, as well as the Shanks nonlinear transformation:

$$
\left[a+\left(a^{2}-b\right) \cdot \varepsilon\right] /\left.(a-b \cdot \varepsilon)\right|_{a^{2}=b}=(1-a \cdot \varepsilon)^{-1} .
$$

The values of the approximation parameters obtained in this case $\left(b=a^{2}\right)$ from (8) are equal to $M=-a, N=-1$, i. e. coincide with the parameters of the right-hand side of (10), the exact expression for the sum of the geometric progression.

\section{Example 1}

Let's compare the error of approximation with the developed method with the error of the well-known Padé approximation method. Let's consider here an example given in [7], where an approximation is given of a fragment of the Maclaurin series (i. e., its first three terms) into which the function $\mathbf{y}(\mathrm{z})=[\ln (1+\mathrm{z})] / \mathrm{z}=1-\mathrm{z} / 2+\mathrm{z}^{2} / 3-\ldots \operatorname{In}[7]$ three Padé approximations are given: [1/0], [0/1] and the most accurate of them $[1 / 1]=(1+z / 6) /(1+2 \cdot z / 3)$, which gives an error of $1 \%$ for $z=1$. If the approximated series is approximated by a new method, for example, within the framework of Newton binomial (in this case, the values of the approximation parameters calculated according to formulas (8): $M=5 / 6$, and $N=3 / 5)$, then let's obtain the approximate formula $y(z)=(1+5 \cdot z / 6)^{-0,6}$, which for $\mathrm{z}=1$ gives an error of less than $0.3 \%$. Thus, it is obvious that the method developed in the paper for approximating the first three terms of the Maclaurin series is more accurate in this case than the known Padé approximation method.

\section{Example 2}

In applications it is often necessary to express the variable of the resulting solution ( $\mathrm{x}$ ) in an explicit form: $\mathrm{x}=\mathrm{f}(\mathrm{y})$. This is the case, for example, in inverse problems in the definition of physical properties. However, in many solutions the variable (x) can't be explicitly expressed, for example, if the solution is obtained in the form $y=[1-\exp (-x)] / x,[10]$. To obtain the explicit expression for $x$, let's use the approximation of the relation $y=f(x)$ in the form of a Newton's binomial. Using the formula (8) for $y(x)$, it is possible to obtain the following approximation:

$$
\mathbf{y}(\mathrm{x})=[1-\exp (-\mathrm{x})] / \mathrm{x} \approx(1+\mathrm{x} / 6)^{-3}
$$

From the found approximation, it is easy to express the variable (x) in the form:

$$
\mathrm{x}=\mathrm{f}(\mathbf{y}) \approx 6 \cdot[\sqrt[3]{(1 / \mathbf{y})-1}]
$$

This approximation has sufficient approximation accuracy in the range (0-2).

And if a more accurate approximation is needed, then for $\mathrm{x} \geq 0$, it is possible to use double approximation here and get its approximation by a more complicated function:

$$
\mathbf{y}(\mathrm{x})=[1-\exp (-\mathrm{x})] / \mathrm{x} \approx \exp (\cos \sqrt{\mathrm{x}-1})
$$

From this approximation, the variable $\mathrm{x}$ can be expressed in a more complicated way:

$$
x=f(y)=\arccos ^{2}(1+\ln \mathbf{y})
$$

This approximation has not only higher accuracy, but also a wider range of applications (0-3).

Sometimes it is necessary to find an approximation for the function $y=x / \ln (1-x)^{-1}$, which describes the dependence of the "efficiency" $y(x)$ in the range $0 \leq x \leq 1$. If we use Newton's binomial as a basis here, we obtain the following approximation

$$
\mathbf{y} \approx(1-x / 1,2)^{3} .
$$




\section{2. Approximation of functions that decompose into a power series in the square of} the variable $x^{2}$

In a number of cases, when solving nonlinear problems by the small parameter method, the result is a fragment of the series in the form of an expansion in even powers of the argument. This is often the case in periodic processes described by trigonometric functions, for example, cosine:

$$
\cos (x)=1-\frac{x^{2}}{2 !}+\frac{x^{4}}{4 !}-\frac{x^{6}}{6 !}+\ldots
$$

In such cases, the fragment of the series is obtained in the following form:

$$
\sum c_{i} x^{i}=1+a x^{2}+b x^{4}+\ldots
$$

To approximate such series, as a basis, let's use functions that decompose into a Maclaurin series with respect to even powers of the argument:

$$
\mathrm{f}^{\mathrm{N}}(\mathrm{x})=1+\mathrm{N} \cdot \mathrm{f}_{(0)}^{\prime \prime} \cdot \frac{\mathrm{x}^{2}}{2 !}+\mathrm{N} \cdot\left[3(\mathrm{~N}-1)\left(\mathrm{f}^{\prime \prime}{ }_{(0)}\right)^{2}+\mathrm{f}_{(0)}^{\mathrm{iv}}\right] \cdot \frac{\mathrm{x}^{4}}{4 !}+\ldots
$$

Carrying out in (17) a change of variable $x=\varepsilon \sqrt{M}$ and equating the coefficients of the corresponding $\varepsilon$ powers of series (11) and (12), let's obtain the system:

$$
\left\{\begin{array}{l}
\mathrm{a}=(\mathrm{M} \cdot \mathrm{N} / 2) \cdot \mathrm{f}_{(0)}^{\prime \prime}, \\
\mathrm{b}=\left(\mathrm{M}^{2} \cdot \mathrm{N} / 24\right) \cdot\left[3(\mathrm{~N}-1) \cdot\left(\mathrm{f}_{(0)}^{\prime \prime}\right)^{2}+\mathrm{f}_{(0)}^{\mathrm{IV}}\right]
\end{array}\right.
$$

Solving the obtained system of equations for the unknown parameters $\mathrm{M}$ and $\mathrm{N}$, let's obtain their expressions through the known coefficients a and $b$ of the series (1) and the derivatives of the function $f_{(x)}$ chosen as the basis for the approximation $f^{\prime}(x)$ and $f^{\prime \prime}(x)$ in point $(0,1)$ :

$$
\begin{aligned}
& \mathrm{N}=\left(\mathrm{a} / \mathrm{f}^{\prime \prime}{ }_{(0)}\right)^{2} \cdot\left[\mathrm{f}^{\mathrm{iv}}{ }_{(0)} / 3-\left(\mathrm{f}^{\prime \prime}{ }_{(0)}\right)^{2}\right] /\left(2 \mathrm{~b}-\mathrm{a}^{2}\right), \\
& \mathrm{M}=2\left(\mathrm{f}^{\prime \prime}{ }_{(0)} / \mathrm{a}\right) \cdot\left(2 \mathrm{~b}-\mathrm{a}^{2}\right) /\left[\mathrm{f}^{\mathrm{iv}}{ }_{(0)} / 3-\left(\mathrm{f}^{\prime \prime}{ }_{(0)}\right)^{2}\right] .
\end{aligned}
$$

\section{Example 3}

If it is necessary to approximate functions that decompose into series in even powers of the argument, it is very simple to use Newton's binomial as a basis. In this case, first we need to change the variable $\varepsilon=\mathrm{x}^{2}$, and then use formulas (8).

For example, for the cosine function: $\cos (\mathrm{x}) \approx\left(1-\mathrm{x}^{2} / 3\right)^{1,5}$. The function $\mathrm{y}=\mathrm{x} / \operatorname{arth}(\mathrm{x})$ that is implicit with respect to $\mathrm{x}$ is also expressed in terms of the square of the variable $\mathrm{x} / \operatorname{arth}(\mathrm{x}) \approx$ $\approx\left(1-13 x^{2} / 15\right)^{1 / 2,6}$. And for the Bessel function of the first kind of order zero, the approximation by the Newton binomial in terms of the square of the variable is very simple: $\mathrm{J}_{0}(\mathrm{x}) \approx\left(1-\mathrm{x}^{2} / 8\right)^{2}$.

A more complicated expression, but less precise, can be obtained by expressing the Bessel function via the cosine function: $\mathrm{J}_{\mathrm{o}}(\mathrm{x}) \approx[\cos (\mathrm{x} \cdot \sqrt{3 / 2})]^{2 / 3}$. However, in this case it is necessary to use the general approach (7).

\section{3. Estimation of the approximation error}

If, as a result of solving a nonlinear problem, a solution is obtained in the form of a fragment of a power series of the first three terms of the Maclaurin expansion, then it is obviously impossible to estimate the error of approximate formulas obtained by this method. But if, as a result of the solution, a fragment is obtained from the first four terms, it becomes possible to estimate the approximation error. There is also this possibility in the other two cases considered above: if necessary, obtain an approximate formula in the case when for an exact function one can't obtain an explicit 
expression of the argument, and also in cases of simplification of very complicated formulas by simple expressions without significant loss of accuracy.

For example, if as a result of solving a nonlinear problem by a small parameter method, a solution is found in the form of a fragment of a power series of the first four terms of the Maclaurin expansion:

$$
\mathbf{y}(\mathbf{x})=1+\mathbf{y}_{(0)}^{\prime} \cdot \frac{\mathbf{x}}{1 !}+\mathbf{y}_{(0)}^{\prime \prime} \cdot \frac{\mathrm{x}^{2}}{2 !}+\mathbf{y}_{(0)}^{\prime \prime \prime} \frac{\mathrm{x}^{3}}{3 !}
$$

then, as a result of approximation for the solution of the problem obtained above, the approximate formula $\mathrm{f}(\mathrm{x})$ (that is, $\mathrm{y}(\mathrm{x}) \approx \mathbf{f}(\mathrm{x})$ ), is obtained by the proposed method, which expansion in the power series has the following form:

$$
\mathbf{f}(\mathrm{x})=1+\mathbf{f}_{(0)}^{\prime} \cdot \frac{\mathrm{x}}{1 !}+\mathbf{f}_{(0)}^{\prime \prime} \cdot \frac{\mathrm{x}^{2}}{2 !}+\mathbf{f}_{(0)}^{\prime \prime \prime} \cdot \frac{\mathrm{x}^{3}}{3 !}+\ldots
$$

In this case, as an accurate estimate of the absolute error $\Delta(\mathrm{x})$ of the approximation, it is possible to use the difference of two series $\mathbf{R}(\mathrm{x})=\mathbf{f}(\mathrm{x})-\mathbf{y}(\mathrm{x})$. Since the first three terms of the series (21) and (20) are respectively equal, the result of the subtraction is also expressed by a power series whose first term is:

$$
\mathbf{R}(\mathrm{x}) \approx \mathrm{X}^{3}\left(\mathbf{f}^{\prime \prime \prime}{ }_{(0)}-\mathbf{y}_{(0)}^{\prime \prime \prime}\right) / 6+\mathrm{X}^{4} \cdot \mathbf{f}_{(0)}^{\mathrm{IV}} / 24+\ldots
$$

As a rough estimate of the absolute error of approximation $\Delta(\mathrm{x})$ in this case, it is possible to use the value of the first term of this series:

$$
\Delta(\mathrm{x}) \approx \mathrm{X}^{3}\left(\mathbf{f}^{\prime \prime \prime}{ }_{(0)}-\mathbf{y}^{\prime \prime \prime}{ }_{(0)}\right) / 6
$$

A similar result can be obtained in the other two cases mentioned, only then the power series $\mathbf{R}(\mathrm{x})$ will have the following form:

$$
\mathbf{R}(\mathrm{x}) \approx \mathrm{X}^{3}\left(\mathbf{f}^{\prime \prime \prime}{ }_{(0)}-\mathbf{y}^{\prime \prime \prime \prime}{ }_{(0)}\right) / 6+\mathrm{X}^{4}\left(\mathbf{f}^{\mathrm{IV}}{ }_{(0)}-\mathbf{y}_{(0)}^{\mathrm{IV}}\right) / 24+\ldots
$$

And as a rough estimate of the relative error of approximation in this case, it is possible to use the expression:

$$
\boldsymbol{\delta}(\mathrm{x}) \approx \mathrm{X}^{3}\left(\mathbf{f}^{\prime \prime \prime}{ }_{(0)}-\mathbf{y}^{\prime \prime \prime}\left({ }_{(0)}\right) /[6 \mathbf{y}(\mathrm{x})]\right.
$$

An analysis of the above approximate estimates of the error in the approximation of functions by the method developed in the article showed that $\Delta(\mathrm{x})$ is proportional to the third power of the variable, that is, $\mathrm{x}^{3}$. And to approximate functions that decompose with respect to even powers of the argument $\Delta(\mathrm{x})$, its proportionality to the sixth power of the argument is obvious, that is, $\mathrm{x}^{6}$.

\section{Discussion of research results}

The method for approximating the sum of a power series developed in the article can also be used in the case of approximation of functions that decompose into a Taylor series, for example, in the cases when $\mathrm{x}_{(0)}=\mathrm{d}$, then the power series has the form:

$$
\mathbf{y}(x)=\mathbf{y}(d)+\mathbf{y}^{\prime}(d) \cdot(x-d)+\mathbf{y}^{\prime \prime}(d) \cdot(x-d)^{2} / 2+\ldots
$$

Normalizing the coefficients of the obtained series (15) by dividing them by the value of $\mathbf{y}$ at the point $\mathrm{d}$ : $\mathrm{y}(\mathrm{d})$ (obviously, the condition: $\mathrm{d}$ : $\mathrm{y}(\mathrm{d})$ easily transforms the series (15) into a 
Maclaurin series. The subsequent sequence of parameter determination in the approximation is described above.

In this case, the choice of a number of proposed approximants is as follows. It is necessary to compare the coefficients of the third (or sixth) degree of the variable expansion of the proposed functions, with the corresponding coefficient for the third (or sixth) power of the variable $\varepsilon$ of the expansion of the original function in a Taylor series. In this case, the criterion for choosing the approximant is, obviously, the minimum of the relative error of approximation of this coefficient.

\section{Conclusions}

For functions decomposing into Maclaurin series, a new analytical method for obtaining approximate formulas in the form of the Nth power of the functions $\mathrm{f}(\mathrm{Mx})$, adopted a priori as a function of the basis of approximation, is developed.

In general terms, expressions for the approximation parameters $(\mathrm{N}$ and $\mathrm{M})$ of the approximate formula are obtained from the coefficients of the first three terms of the expansion of the function; therefore, even a small fragment of the series found as a result of solving the nonlinear problem by the small parameter method can serve as the initial function.

The developed method can also be used to obtain approximate formulas in cases where it is impossible to express the argument explicitly from exact functions, and also in cases where it is necessary to simplify complex formulas by simple expressions without significant loss of accuracy.

The error of approximations is analyzed and expressions are obtained for estimating the absolute and relative error of approximation.

\section{References}

[1] Koul, Dzh. (1972). Metody vozmushheniy v prikladnoy matematike. Moscow: MIR, 275.

[2] Van-Dayk, M. (1967). Metody vozmushheniy v dinamike zhidkosti. Moscow: MIR, 311.

[3] Popov, V. A., Tesler, G. S. (1980). Priblizhenie funktsiy dlya tekhnicheskikh prilozheniy. Kyiv: Naukova dumka, 353.

[4] Krylov, V. I. (1988). Matematicheskiy analiz. Uskorenie skhodimosti. Minsk: N i T, 175.

[5] Baker, G. A. Jr. (1965). The theory and application of the Pade approximant method. Advances in Theoretical Physics, 1, 1-58.

[6] Baker, G. A. Jr. (1975). Essentials of Pade approximants. New York: Academic Press, 318.

[7] Baker, G. A. Jr., Graves-Morris, P. (1981). Pade Approximants. Addison-Wesley Publishing Co.

[8] Vinogradov, V. N., Gay, E. V., Rabotnov, N. S. (1987). Analiticheskaya approksimatsiya dannykh v yadernoy i neytronnoy fizike. Moscow: Energoatomizdat, 128.

[9] Ludanov, K. I. (1984). Metod approksimatsii summy stepennogo ryada po ego pervym trem chlenam. Uk-84, Dep. № 763 v UkrNIINTI. Kyiv, 7.

[10] Fisher, M. E. (1965). The nature of critical points. Boulder: University of Colorado Press, 159. 